\title{
Non-Coherent Code Acquisition in the Multiple Transmit/Multiple Receive Antenna Aided Single- and Multi-Carrier DS-CDMA Downlink
}

\author{
SeungHwan Won and Lajos Hanzo, Fellow, IEEE
}

\begin{abstract}
We analyse the characteristics of the Non-Coherent (NC) Multiple Transmit/Multiple Receive (MTMR) antenna aided Multi-Carrier (MC) DS-CDMA downlink employing a serial search based acquisition scheme, when communicating over spatially uncorrelated Rayleigh channels. The associated Mean Acquisition Time (MAT) performance trends are characterised as a function of both the number of antennas and that of the number of subcarriers. It is shown that the employment of both multiple transmit antennas and multiple subcarriers is typically detrimental in terms of the achievable NC acquisition performance, while that obtained by exploiting multiple receive antennas is always beneficial, regardless whether single-path or multi-path scenarios are considered. Based on our results justified by information theoretic considerations, our acquisition design guidelines are applicable to diverse NC MTMR antenna aided scenarios.
\end{abstract}

Index Terms-MC-DS-CDMA, non-coherent, transmit/receive/ frequency diversity.

\section{INTRODUCTION}

$\mathbf{E}$ MPLOYING both multiple transmit antennas and multiple subcarriers in the DownLink (DL) of wireless systems exhibits an attractive technique of reducing the detrimental effects of time-variant multi-path fading environments, while maintaining an increased design flexibility [1],[2]. The combination of Single-Carrier (SC) CDMA and OFDM has drawn substantial research attention [2],[3]. In inter-cell synchronous CDMA systems the Mobile Station (MS)'s receiver must be capable of synchronously aligning a locally generated PseudoNoise (PN) code with the desired user's PN sequence. Substantial research efforts have been devoted to the design of code acquisition techniques for singleinput single-output systems [3] with the aim of minimising the MAT. The serial acquisition performance of MC-DSCDMA has been analysed in [4]. However, since no indepth analysis of the effects of diversity gain on serial search based spreading code acquisition schemes designed for the MTMR antenna aided MC-DS-CDMA DL is available in the literature, carrying out this analysis is the objective of this letter. Against this backcloth, we set out to analyse a serial search based NC acquisition scheme designed for the MTMR antenna aided MC-DS-CDMA DL. More explicitly, we quantify both the correct detection probability $\left(P_{D}\right)$ and the false alarm probability $\left(P_{F}\right)$ as well as the MAT versus the Signal-to-Interference plus Noise Ratio (SINR) per chip

Manuscript received February 25, 2006; revised February 24, 2007; accepted September 8, 2007. The associate editor coordinating the review of this paper and approving it for publication was M. Sawahashi.

The authors are with the University of Southampton, UK (e-mail: $\{1 \mathrm{~h}$, shw04r\}@ecs.soton.ac.uk).

Digital Object Identifier 10.1109/TWC.2007.06026.
$\left(E_{c} / I_{0}\right)$ performance parameterised by both the number of antennas and that of the subcarriers.

\section{Correct Detection And False Alarm PROBABILITY}

The DL transmitted signal of the Base Station (BS) having $P$ transmit antennas and $U$ subcarriers can be expressed as

$$
\sum_{m=1}^{P} \sum_{u=1}^{U}\left[\sqrt{\frac{E_{c}}{P T_{c}}} b(t) c(t) w_{m}(t) \cdot e^{\left(2 \pi f_{u} t+\phi_{u}\right)}\right],
$$

where $m=1, \ldots, P$ indicates the number of transmit antennas, $u=1, \ldots, U$ is the number of subcarriers, $b(t)$ represents the pilot data sequence assuming a value of binary '1' [5], $c(t)$ denotes a common PN sequence having a cell-specific codephase offset, $w_{m}(t)$ identifies the specific Walsh code assigned to the $m^{\text {th }}$ transmit antenna, $E_{c}$ denotes the pilot signal energy per PN code chip, $T_{c}$ indicates the chip duration, $f_{u}$ is the $u^{t h}$ subcarrier frequency and $\phi_{u}$ denotes the $u^{\text {th }}$ subcarrier phase of the modulator. In the MC-DS-CDMA system considered the input bit sequence is Serial-to-Parallel (SP) converted and each of the parallel sequences is transmitted on a separate subcarrier. Furthermore, $T_{b}$ represents the bit duration of the data sequence before SP conversion, while $T_{s}$ indicates the symbol duration after SP conversion. Accordingly, we have $T_{s}=U \cdot T_{b}$. The total allocated power is equally shared by both the $P$ transmit antennas and the $U$ subcarriers. The spacing of both the multiple transmit antennas at the BS and the multiple receive antennas at the MS are assumed to be $10 \lambda$ and $0.5 \lambda$, respectively. It is also assumed that the BS antennas have a high elevation. The Spreading Factor (SF) of the MC-DS-CDMA system's subcarriers is $S F_{0}=T_{s} / T_{c}$, while $S F_{1}=T_{b} / T_{c 1}$ denotes the SF of a corresponding SC-DS-CDMA system, having a chip duration of $T_{c 1}$. For simplicity, we postulate in our forthcoming analysis that the main spectral lobes of two adjacent subcarriers do not overlap in the MC-DS-CDMA system considered here [4]. Furthermore, we assume that each subcarrier signal occupies an identical bandwidth and the total bandwidth is equally divided among the $U$ number of subcarriers. Therefore, the relationships of $T_{c}=U \cdot T_{c 1}$ and $S F_{1}=S F_{0}$ hold since we have $T_{s}=U \cdot T_{b}$. According to the above assumption, both the MC- and the corresponding SC-DS-CDMA systems have the same bandwidth, as suggested in [4], which allows their direct comparison in our forthcoming discourse. We also assume that a finite-length tapped delay line channel model generates $L$ Rayleigh-faded multi-path signals, each arriving with a time delay $\tau_{l}$ having a tap spacing of one chipduration [6],[7], where $l=1, \ldots, L$ is the number of multi-path components. The signal of the MTMR antenna aided MC- 


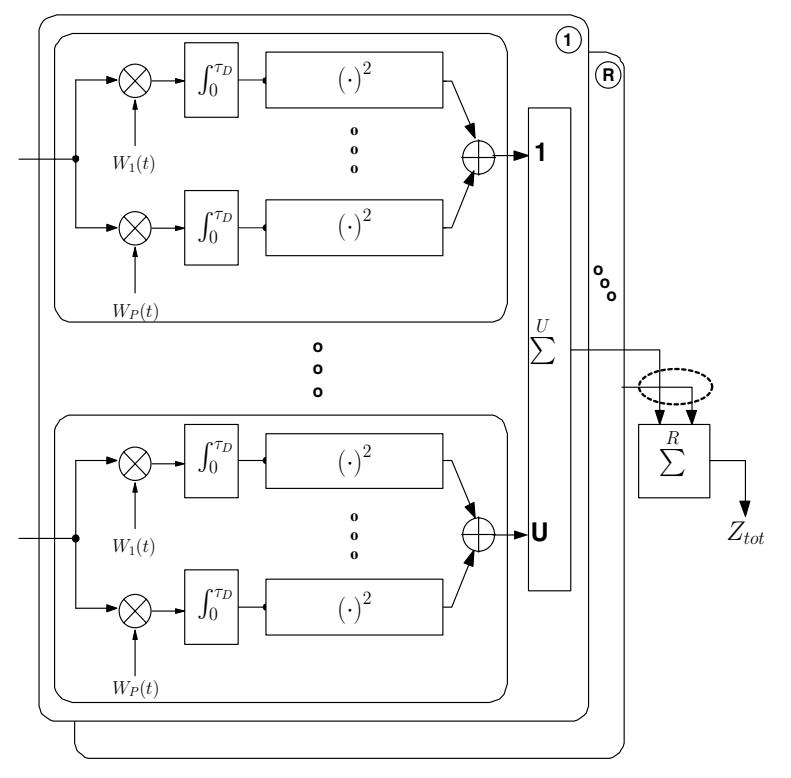

Fig. 1. Receiver schematic of a MC-DS-CDMA code acquisition employing both $R$ receive antennas and $U$ subcarriers.

DS-CDMA DL received over the multi-path Rayleigh fading channel considered may be formulated as [8]

$$
\begin{aligned}
r_{\text {tot }}(t)= & \sum_{(l, m, n, u)=1}^{(L, P, R, U)}\left[\alpha_{(l, m, n, u)} \sqrt{\frac{E_{c}}{P T_{c}}} b\left(t^{\prime}\right) c\left(t^{\prime}\right)\right. \\
& \left.\cdot w_{m}\left(t^{\prime}\right) e^{\left(2 \pi f_{u} t+\phi_{(l, m, n, u)}\right)}+I_{(l, m, n, u)}(t)\right],
\end{aligned}
$$

where $t^{\prime}$ represents $t+d T_{c}+\tau_{l}, n=1, \ldots, R$ is the number of receive antennas, $\alpha_{(l, m, n, u)}$ represents the complex-valued envelope of the $(l, m, n, u)^{t h}$ received signal path obeying the Rayleigh distribution, whilst $d$ is the code phase offset with respect to the phase of the local code ${ }^{1}$. Furthermore, $I_{(l, m, n, u)}(t)$ is the complex-valued Additive White Gaussian Noise (AWGN) having a double-sided power spectral density of $I_{0}$, which contaminates the $(l, m, n, u)^{t h}$ path. The total amount of channel-induced impairments imposed on the DL are constituted by the superposition of the background noise, plus the serving-cell interference imposed by both the multi-path signals and the other users as well as the othercell interference plus the inter-subcarrier interference. Further details on the calculation of the total interference may be found in [5],[9]. Fig.1 depicts the receiver's schematic designed for our MC-DS-CDMA code acquisition scheme using MTMR antennas, which generates the decision variable by accumulating $(P \cdot R \cdot U)$ number of independently faded signals observed over a time interval, where $\tau_{D}=N \cdot T_{c}$ indicates the integral dwell time and $N$ represents the number of chips accumulated over the duration of $\tau_{D}$. The final decision variable of the $l^{t h}$ path may be expressed with the aid of the procedures suggested in [8] as follows

$$
1 \sum_{(l, m, n, u)=1}^{(L, P, R, U)} \text { denotes } \sum_{l=1}^{L} \sum_{m=1}^{P} \sum_{n=1}^{R} \sum_{u=1}^{U} \text {. }
$$

$$
\begin{aligned}
Z_{\text {tot }} & =\sum_{(m, n, u)=1}^{(P, R, U)} Z_{k(l, m, n, u)} \\
& =\sum_{(m, n, u)=1}^{(P, R, U)}\left\|\frac{1}{\sqrt{2}} \cdot\left(\sqrt{\gamma} \cdot S_{k(l, m, n, u)}+I_{k(l, m, n, u)}\right)\right\|^{2}(3)
\end{aligned}
$$

where $\gamma$ represents $\frac{4 E_{c}}{N I_{0} P}, k$ denotes the $k^{t h}$ chip's sampling instant, $S_{k(l, m, n, u)}$ is assumed to be deterministic, while $I_{k(l, m, n, u)}$ is the complex-valued AWGN having zero means and variances of $\sigma^{2}=2$ for both their real and imaginary parts, as shown in [8]. Furthermore, $\|\cdot\|^{2}$ represents the Eucledian norm of the complex-valued argument and $1 / \sqrt{2}$ is a normalisation factor regarding the noise variance. It is worth noting that the outputs of the squaring operation invoked for both the in-phase and the quadrature branch of $Z_{k(l, m, n, u)}$ in Fig.1 are modelled as the square of the Gaussian random variable, respectively. Accordingly, the decision variable $Z_{k(l, m, n, u)}$ of each path obeys a non-central chi-square Probability Density Function (PDF) with two degrees of freedom [7] and having a non-centrality parameter of $\lambda_{x}$, which is either $\frac{2 N}{P}\left(\frac{E_{c}}{I_{0}}\right)$ for the hypothesis of the desired signal being present $(x=1)$ or $\frac{2}{N P}\left(\frac{E_{c}}{I_{0}}\right)$ for it being absent $(x=0)$ [8]. This PDF is given by [6]

$$
f_{Z_{k(l, m, n, u)}}\left(z \mid H_{x}\right)=\frac{1}{2} \cdot \exp \left[-\frac{\left(z+\lambda_{x}\right)}{2}\right] \cdot \mathcal{I}_{0}\left(\sqrt{z \cdot \lambda_{x}}\right),
$$

where $z \geq 0, x=0$ or 1 and $\mathcal{I}_{0}(\cdot)$ is the $z$ ero ${ }^{t h}$-order modified Bessel function. Our aim is now that of expressing the PDF of the desired user's signal at the output of the acquisition scheme conditioned on the presence of the desired signal in $f_{Z_{\text {tot }}}\left(z \mid H_{x}\right)$ derived for transmission over a spatially uncorrelated Rayleigh channel. In this scenario $E_{c}$ is multiplied by the square of the Rayleigh-distributed fading amplitude, $\beta$, which exhibits a chi-square distribution having two degrees of freedom and it is hence expressed as $f(\beta)=\frac{e^{-\beta / \sigma^{2}}}{\sigma^{2}}$, where $\sigma^{2}$ is the variance of the constituent Gaussian distribution. Then the average pilot signal energy $\overline{E_{c}}$ per PN code chip can be expressed as $\overline{E_{c}}=\bar{\beta} E_{c}=\sigma^{2} E_{c}$ [5]. Hence first the PDF $f_{Z_{k(l, m, n, u)}}\left(z \mid H_{x}, \beta\right)$ corresponding to $\beta$ conditioned on the hypothesis of the desired signal being transmitted over an AWGN channel having this specific SINR is weighted by the probability of occurrence $f(\beta)$ of encountering $\beta$, as quantified by the PDF. The resultant product is then averaged over its legitimate range of $-\infty \sim \infty$, yielding:

$$
\begin{aligned}
f_{Z_{k(l, m, n, u)}}\left(z \mid H_{x}\right)= & \int_{-\infty}^{\infty} f(\beta) \cdot f_{\left.Z_{k(l, m, n, u}\right)}\left(z \mid H_{x}, \beta\right) d \beta(5) \\
= & \int_{0}^{\infty}\left(\frac{e^{-\beta / \sigma^{2}}}{\sigma^{2}}\right) \cdot \frac{\exp \left[-\left(z+\beta \lambda_{x}\right) / 2\right]}{2} \\
& \cdot \mathcal{I}_{0}\left(\sqrt{\beta \lambda_{x} z}\right) d \beta \\
\equiv & \frac{\exp \left[-z /\left(2+\overline{\lambda_{x}}\right)\right]}{\left(2+\overline{\lambda_{x}}\right)}
\end{aligned}
$$

where the effects of both timing errors and frequency mismatch are encapsulated by the definition of $\overline{\left(E_{c} / I_{0}\right)^{\prime}}$. In the spirit of [5], $\overline{\left(E_{c} / I_{0}\right)^{\prime}}$ is defined as $\overline{\left(E_{c} / I_{0}\right)^{\prime}}=$ $\overline{\left(E_{c} / I_{0}\right)} \cdot \operatorname{sinc}^{2}\left(\frac{\tau}{T_{c}}\right) \cdot \operatorname{sinc}^{2}\left(N \Delta f_{t} T_{c}\right)$, where the second multiplicative term of this definition represents the square of 
the autocorrelation function of the timing error, $\tau$, while the third multiplicative term of the definition quantifies the signal energy reduction expressed as a function of the total frequency mismatch, $\Delta f_{t}$ after the squaring operation seen in Fig.1. The corresponding noncentrality parameter, $\overline{\lambda_{x}} \equiv \lambda_{x} \sigma^{2}$ is either $\frac{2 N}{P}\left(\frac{E_{c}}{I_{0}}\right)^{\prime}$ for the hypothesis of the desired signal being present $(x=1)$ or $\frac{2}{N P}\left(\frac{\overline{E_{c}}}{I_{0}}\right)^{\prime}$ for it being absent $(x=0)$. For notational convenience we also define $\mu_{x}=\left(2+\overline{\lambda_{x}}\right)$, which represents a new biased noncentrality parameter. Further details on the related calculations can be found in [5],[8]. Finally, we arrive at the PDF of $Z_{k(l, m, n, u)}$ conditioned the presence of the desired signal in the form of:

$$
f_{Z_{k(l, m, n, u)}}\left(z \mid H_{x}\right)=\frac{1}{\mu_{x}} e^{-z / \mu_{x}} .
$$

Since the decision variable $Z_{t o t}$ is constituted by the sum of $(P \cdot R \cdot U)$ number of independent variables, which has a PDF given by Eq.8, we can derive the Laplace transform of each by raising them to the $(P \cdot R \cdot U)^{t h}$ power and then performing the inverse transform in order to generate the resultant PDF [5], leading to:

$$
f_{Z_{t o t}}\left(z \mid H_{x}\right)=\frac{z^{(P R U-1)} e^{-z / \mu_{x}}}{\Gamma(P R U) \cdot \mu_{x}^{P R U}}
$$

where $\Gamma(\cdot)$ is the Gamma function. Finally, the probability of correct detection or false alarm corresponding to $x=1$ or 0 , respectively, is obtained as

$$
\begin{aligned}
\left.P\right|_{x=1 \text { or } 0} & =\int_{\theta}^{\infty} f_{Z_{\text {tot }}}\left(z \mid H_{x}\right) d z \\
& =\exp \left(-\frac{\theta}{\mu_{x}}\right) \cdot \sum_{k=0}^{(P R U-1)} \frac{\left(\theta / \mu_{x}\right)^{k}}{k !},
\end{aligned}
$$

where $\theta$ is a threshold value.

It is worth noting that the PN sequence's chip duration $T_{c 1}$ of the SC-DS-CDMA system is $U$ times lower than that of the MC-DS-CDMA arrangement, namely, $T_{c 1}=T_{c} / U$. This is because given the same allocated bandwidth and the same total transmitted energy per chip, the bandwidth of the SC-DSCDMA signal is $U$ times higher than that of the subcarrier signals in the MC-DS-CDMA system using $U$ subcarriers. Moreover, for the sake of maintaining a constant integral dwell time of $\tau_{D}$, the chip energy accumulated by the SCDS-CDMA receiver during the period of $\tau_{D}$ is $U$ times higher than that collected by the MC-DS-CDMA correlator of each subcarrier, since the number of chips within the period of $\tau_{D}$ is $U$ times higher for the SC-DS-CDMA system than that of the MC-DS-CDMA system [4],[10].

\section{MAT ANALYSis of CODE ACQUiSition}

The MAT of a single-antenna aided code acquisition scheme was analysed in [5],[9]. The main difference between a singleantenna and a multiple-antenna assisted MC-DS-CDMA system manifests itself in terms of deriving the correct detection and the false alarm probability as a function of both the number of the antennas and that of the subcarriers. We will commence our discourse by analysing the MAT performance of a code acquisition scheme employing Double Dwell Serial Search (DDSS) [9]. We assume that in each chip duration
$T_{c}, \alpha$ number of correct timing hypotheses are tested, which are spaced by $T_{c} / \alpha$. Hence the total uncertainty region is increased by a factor of $\alpha$. Moreover, as mentioned in Section II, when the $L$ multi-path signals arrive with a time delay $\tau_{l}$ having a tap spacing of one chip-duration, then the relative frequency of the signal being present is increased $L$-fold. The required transfer functions [5],[9], are defined as follows. $H_{M}(z)$ represents the overall miss probability of a search run carried out across the entire uncertainty region, while $H_{0}(z)$ indicates the absence of the desired user's signal at the output of the acquisition scheme. The related processes are detailed for DDSS in [9]. The exact MAT formula can be simplified, if $\nu$ is significantly higher than the number of the entire successful detection states [7], where $\nu$ represents the total number of states to be searched. In order to simplify our numerical performance analysis, we adopted the approximation of the exact MAT expression proposed in [7]. Then, it may be shown that the generalised expression derived for computing the MAT of the serial search based code acquisition scheme is given by [7]:

$$
E\left[T_{A C Q}\right] \approx \frac{\left(1+H_{M}(1)\right) \cdot H_{0}{ }^{\prime}(1)}{2 \cdot\left(1-H_{M}(1)\right)} \cdot\left(\nu \cdot \tau_{D 1}\right),
$$

where $\left.H_{x}^{\prime}(z)\right|_{x=M \text { or } 0}$ is a derivative of $\left.H_{x}(z)\right|_{x=M \text { or } 0}$ and $\tau_{D 1}$ denotes the $1^{s t}$ dwell time. Since each resolvable path contributes two successful hypotheses and because the average correct detection probability associated with these two hypotheses is the same, the overall miss probabilities of the DDSS scheme may be expressed as:

$H_{M}(1)=\prod_{l=1}^{L} \prod_{\zeta=1}^{\alpha}\left[\left(1-P_{D 1(l, \zeta)}\right)+P_{D 1(l, \zeta)} \cdot\left(1-P_{D 2(l, \zeta)}\right)\right]^{2}$,

where $\left.P_{D x(l, \zeta)}\right|_{x=1, \text { or } 2}$ are the correct detection probability of both the search and the verification modes of the DDSS arrangements, respectively. $P_{D x(l, \zeta)}$ is given by Eq.11, provided that the condition of the signal being present is satisfied. The $H_{0}{ }^{\prime}(1)$ value of the DDSS scheme is expressed as:

$$
H_{0}{ }^{\prime}(1)=1+m \cdot P_{F 1}+K \cdot P_{F 1} \cdot P_{F 2},
$$

where $m$ represents the exponent of $z$ in the verification mode and $K$ denotes the false locking penalty factor expressed in terms of the number of chip-durations required by an auxiliary device for recognising that the code-tracking loop is still unlocked. Furthermore, $\left.P_{F x}\right|_{x=1, \text { or } 2}$ represent the false alarm probability in both the search and in the verification mode of the DDSS scheme, respectively. Similarly, $P_{F_{x}}$ is given by Eq.11, provided that the condition of the signal being absent is satisfied.

\section{Numerical System Performance Results}

We will characterise the MAT performance of the MTMR antenna aided MC-DS-CDMA code acquisition scheme of Fig.1. Our performance comparison between the SC-DSCDMA system $(U=1)$ and the MC-DS-CDMA $(U=4)$ system using different number of subcarriers is based upon the assumptions that these systems have the same total transmitted energy per chip. Furthermore, it is assumed that $\tau_{D}$ is the same for all the scenarios considered here. In Table I we 
TABLE I

MAXIMUM SINR DEGRADATION INFLICTED BY BOTH THE DOPPLER SHIFT AND THE CLOCK DRIFT ASSOCIATED WITH THE COHERENT INTEGRATION INTERVAL OF $N$ CHIP DURATIONS AT A CARRIER FREQUENCY OF $0.9 \mathrm{GHZ}$ AS A FUNCTION OF THE NUMBER OF SUBCARRIERS $(U=1$ AND 4$)$

\begin{tabular}{|l|l|l|l|l|l|}
\hline N(Chips): U=1 & $\mathbf{1 2 8}$ & 256 & $\mathbf{5 1 2}$ & 768 & 1024 \\
\hline N(Chips): U=4 & $\mathbf{3 2}$ & 64 & $\mathbf{1 2 8}$ & 192 & 256 \\
\hline Degradation $(\mathrm{dB})$ & $\mathbf{0 . 0 4 9}$ & 0.195 & $\mathbf{0 . 7 9 2}$ & 1.827 & 3.371 \\
\hline
\end{tabular}

outlined the maximum SINR degradation imposed by both the Doppler shift and the clock-drift-induced frequency mismatch between the transmitter and receiver in conjunction with the coherent integration interval of $\tau_{D}$ durations, as seen in Fig.1. The length of the PN sequence in our system was assumed to be $\left(2^{15}-1\right) \cdot T_{c 1}\left(\right.$ or $\left.T_{c}\right)$, where the chip-durations employed here for $U=1$ and 4 are $T_{c 1}=1 / 2.4576 \mu s$ and $T_{c}=1 / 0.6144 \mu s$, respectively. Based on [2] when communicating over various fading channels having delay spreads in the range of $[0.1 \mu \mathrm{s}, 3 \mu \mathrm{s}]$, the fading processes of the adjacent subcarriers in our MC-DS-CDMA system are considered to be uncorrelated. The numbers of chips over which the integrator of Fig.1 sums the $(\cdot)^{2}$ envelope detector's output in both the search and the verification modes of DDSS are assumed to be 128 chips and 512 chips in the SC scheme of $U=1$ as well as 32 chips and 128 chips in the $U=4$ senarios, respectively. These optimised parameter values were calculated by using Eqs.11 and 12 as well as Eq.(3.7) of [5], which were provided for quantifying the performance degradation owing to both the Doppler shift and the frequency mismatch encountered. The spreading factor of the Walsh code to be acquired was selected to be 128 . The frequency mismatch was assumed to be $1000 \mathrm{~Hz}$ [5], while the carrier frequency was $0.9 \mathrm{GHz}$. As an example of a high mobile speed, it is reasonable to postulate $160 \mathrm{~km} / \mathrm{h}$. We also assumed that the sampling inaccuracy caused by having a finite, rather than infinitesimally low search step size of $\Delta=1 / 2 T_{c}$ was $-0.91 \mathrm{~dB}$, which is a typical value for the search step size [5],[9]. The total uncertainty region of code acquisition was assumed to entail 65534 hypotheses. In the spirit of [9], the false locking penalty factor was assumed to be 1000 chipdurations. Finally, both single-path and multi-path scenarios were considered. In the $U=1$ scenario both a single-path and three paths arriving with a relative time delay of one chip and having a $3 \mathrm{~dB}$ lower magnitude for the first received path as well as $6 \mathrm{~dB}$ lower for the second and the third received paths, respectively, compared to the Line-Of-Sight path of a singlepath scenario, were assumed to be present in a given search window. On the other hand, in the $U=4$ scenario, only a single received signal path is encountered in a given search window. All the performance curves have been obtained at the value of $E_{c} / I_{0}=-16 \mathrm{~dB}$. This is a threshold value in the range of 'finger locking', which may be considered to be the range between $E_{c} / I_{0}=-17$ and $-13 \mathrm{~dB}[11]$.

Fig. 2 characterises the MAT versus $E_{c} / I_{0}$ performance of DDSS for the SC-DS-CDMA code acquisition arrangement as a function of the number of transmit antennas for $P=1,2$ as well as 4 and that of the number of receive antennas for the specific values of $R=1$ and 4 . In the results of Figs. 2 and

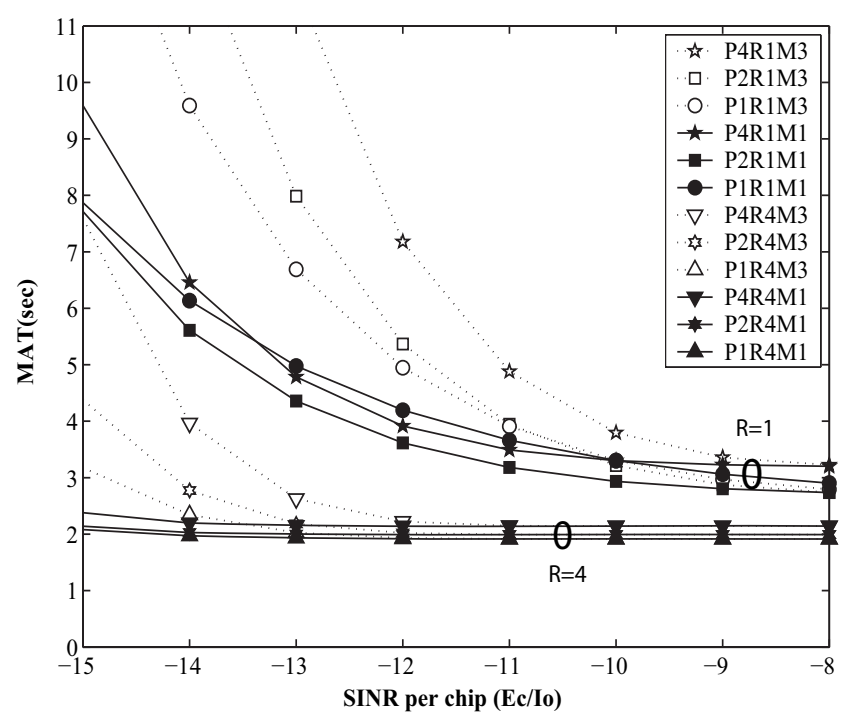

Fig. 2. MAT versus $E_{c} / I_{0}$ performance of the DDSS-aided SC-DS-CDMA code acquisition scheme parameterised with both the number of transmit and receive antennas.

3 , the solid lines indicate a single-path scenario (denoted as $M 1$ in Figs.2 and 3), whereas the dotted lines represent the scenario of receiving three paths (denoted as M3 in Fig.2). It is worth mentioning that although not explicitly shown in Figs. 2 and 3 for avoiding obfuscating details, the operating range of $R=2$ receive antennas was found to be between that corresponding to the $R=1$ and $R=4$ receive antenna scenarios. As the number of transmit antennas was decreased, all the curves seen in Fig. 2 illustrated an improved MAT peformance for the systems exploiting $R=4$ receive antennas in both the single-path and multi-path scenarios. Similar trends were observed also in the $R=1$ receive antenna aided multipath scenario, except for $R=1$ in the specific $E_{c} / I_{0}$ range of the single-path system shown in Fig.2. To elaborate on the above fact a little further, the ' $P 2 R 1^{\prime}$ and ' $P 4 R 1^{\prime}$ scheme communicating over a single-path channel exhibits a better MAT peformance in comparison to the ' $P 1 R 1^{\prime}$ arrangement across the specific $E_{c} / I_{0}$ range considered. In other words, this clearly implies that the scheme employing $R=1$ receive antenna benefits from a higher diversity gain in the SC-DSCDMA scenario having a sufficiently wider bandwidth. By contrast, even though the number of the successfully detected states was increased by a factor of $L=3$, the MAT performance of the multi-path scenario became worse than that of the single-path one. This is because a low 'per-path-power' would lead to a low acquisition performance in the multi-path scenario. Furthermore, the peformance degradation imposed by employing multiple transmit antennas becomes more drastic in the low $E_{c} / I_{0}$ range, as the number of transmit antennas is increased in the scenario. In case of employing both multiple transmit and multiple receive antennas, similar trends are observable, although using two or four receive antennas has the potential of mitigating the associated acquisition performance degradation imposed by the low per-branch $E_{c} / I_{0}$ values associated with the employment of multiple transmitters. In the case of $R=4$ receivers the performance degradations imposed by multiple paths become significantly lower than those in the 


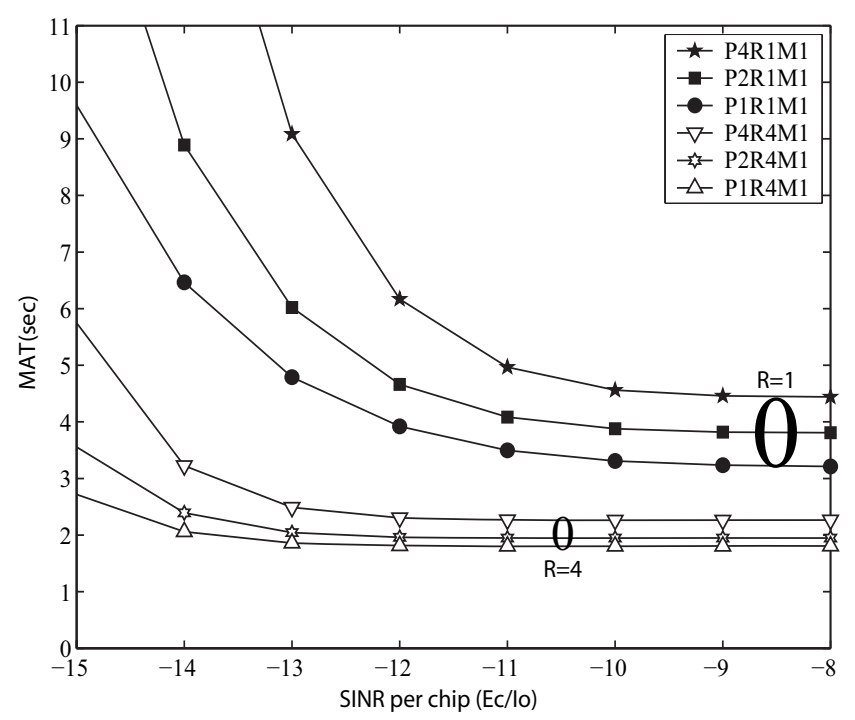

Fig. 3. MAT versus $E_{c} / I_{0}$ performance of the DDSS-aided MC-DS-CDMA code acquisition scheme parameterised with both the number of transmit and receive antennas for $U=4$ subcarriers.

$R=1$ receive antenna scenario, because the receive diversity gain is already sufficiently high for achieving a near-Gaussian MAT-performance, provided that an $E_{c} / I_{0}$ in excess of $12 \mathrm{~dB}$ is maintained for both the single-path and multi-path scenarios. The $R=4$ and $R=1$ scenarios might be viewed as practical upper and lower bounds of the achievable MAT performances encountered. On the other hand, Fig.3 illustrates the achievable MAT versus $E_{c} / I_{0}$ performance of the DDSSaided MC-DS-CDMA code acquisition scheme parameterised with the number of antennas, when using $U=4$ subcarriers. In the case of the MC-DS-CDMA system, the scheme has an unnecessarily high diversity order, which is determined by the number of subcarriers used. It is also assumed that the total transmitted energy per chip is the same in all the scenarios considered. Accordingly, the effect of the inherent frequency diversity is the same as that of the multiple transmit antenna aided diversity. This fact indicates that employing multi-carrier transmissions based on the DS-CDMA principle leads to exactly the same detrimental effect on the achievable MAT performance as that imposed by employing the multiple transmit antennas. The results of Fig. 3 are parameterised by both the number of transmit antennas for $P=1,2$ as well as 4 and by the number of receive antennas for $R=1$ as well as 4 . As the number of transmit antennas is decreased, all the curves seen in Fig.3 indicate an improved MAT peformance. This trend explicitly illustrates that the performance of the MCDS-CDMA acquisition scheme becomes significantly worse than that of SC-DS-CDMA encountering a single-path. This is a consequence of both the low per-antenna power imposed by employing a transmit diversity gain and the low persubcarrier power imposed by using a frequency diversity gain. To interpret the above results a little further, a low level of perbranch and/or per-subcarrier received signal strength would lead to a low acquisition performance, despite achieving a high transmit- and frequency-diversity gain, as a result of the insufficiently high signal strength per transmit antenna and per subcarrier. When comparing the results of Figs.2 and 3, the performance of the MC-DS-CDMA code acquisition scheme encountering a single-path is a bit better than that of the SCDS-CDMA code acquisition scheme encountering three paths. It also suggests that a low level of per-path received signal strength would result in a low acquisition performance, despite having a four times higher number of accumulated chips over the same integral dwell time.

The main reasons for the above-mentioned performance trends may also be further justified by the information theoretic considerations derived for NC MTMR antenna aided scenarios in [10],[12],[13]. Finally, when considering the design of MTMR antenna aided code acquisition schemes, the following guidelines may be inferred:

a) Using multiple transmit antennas typically leads to an MAT performance degradation, except for specific scenarios, when encountering a single-path environment, also depending on the allocated bandwidth. Using a relatively low number of chips, over which integration or accumulation is carried out, imposes further limits on the attainable benefits of MTMR antennas. Furthermore, in the multi-path scenarios considered all the results fail to show a transmit diversity gain. Therefore, activating only a single transmit antenna might be recommended for the sake of maximising the achievable MAT performance of the code acquisition scheme investigated.

b) Employing multiple receive antennas increases the achievable receiver diversity gain and has the potential of compensating for the MAT degradation imposed by the low per-branch power of both multiple transmitters and multiple subcarriers.

c) In order to acquire the exact timing information of the received paths without any potential performance degradation that might be imposed on NC MTMR antenna aided scenarios, specificially designed preambles, such as that of the primary synchronization channel of W-CDMA [14] combined with time-switched transmit diversity might be recommended, which is capable of achieving a diversity gain with the aid of a single transmit antenna [12]. The conventional pilot channel may also be used for other purposes, such as frequency error correction and channel estimation so as to support coherent MTMR antenna aided scenarios.

\section{CONCLUSION}

We analysed the MTMR antenna aided code acquisition performance of the inter-cell synchronous SC- and MC-DSCDMA DL. Unexpectedly, our results suggest that increasing both the number of transmit antennas and that of the subcarriers in the MTMR antenna aided MC-DS-CDMA DL results in amalgamating the low-energy, noise-contaminated signals of both the transmit antennas and the subcarriers. Furthermore, communicating in a multi-path scenario also degrades the attainable performance. Based on the above-mentioned results, which are also corroborated by the information theoretic considerations of [12], our acquisition design guidelines are applicable to diverse NC MTMR antenna aided scenarios. Hence our future research will be focused on specifically designing iterative turbo acquisition schemes for NC MTMR antenna aided MC transmission systems [3]. 


\section{ACKNOWLEDGEMENT}

The financial support of the Ministry of Information and Communication (MIC), Republic of Korea and the European Union under the auspices of the Phoenix and Newcom projects and that of the EPSRC is gratefully acknowledged.

\section{REFERENCES}

[1] D. Gesbert, M. Shafi, D. S. Shiu, P. J. Smith, and A. Naguib, "From theory to practice: an overview of MIMO space-time coded wireless systems," IEEE J. Sel. Areas Commun., vol. 21, no. 3, pp. 281-302, 2003.

[2] L.-L. Yang and L. Hanzo, "Multicarrier DS-CDMA: a multiple access scheme for ubiquitous broadband wireless communications," IEEE Commun. Mag., vol. 41, no. 10, pp. 116-124, 2003.

[3] L. Hanzo, L.-L. Yang, E.-L. Kuan, and K. Yen, Single- and MultiCarrier DS-CDMA. John Wiley \& Sons, 2003.

[4] L.-L. Yang and L. Hanzo, "Serial acquisition performance of singlecarrier and multicarrier DS-CDMA over Nakagami-m fading channels," IEEE Trans. Wireless Commun., vol. 1, no. 4, pp. 692-702, 2002.

[5] A. J. Viterbi, CDMA: Principles of Spread Spectrum Communication, Chapter 3. Addison-Wesley, 1995.

[6] J. G. Proakis, Digital Communications, 4th ed., Chapter 2. McGrawHill, 2001, pp. 17-79.
[7] L.-L. Yang and L. Hanzo, "Serial acquisition of DS-CDMA signals in multipath fading mobile channels," IEEE Trans. Veh. Technol., vol. 50, no. 2, pp. 617-628, 2001.

[8] J.-C. Lin, "Differentially coherent PN code acquisition with full-period correlation in chip-synchronous DS/SS receivers," IEEE Trans. Commun., vol. 50, no. 5, pp. 698-702, 2002.

[9] H. R. Park, "Performance analysis of a double-dwell serial search technique for cellular CDMA networks in the case of multiple pilot signals," IEEE Trans. Veh. Technol., vol. 48, no. 6, pp. 1819-1830, 1999.

[10] S. Ray, M. Medard, and L. Zheng, "On MIMO capacity in the ultrawideband regime, signals, systems and computers," in Conference Record Thirty-Eighth Asilomar Conference, vol. 2, 2004, pp. 1516-1520.

[11] C. Noblet, M. Fadridis, and R. Owen, "Downlink transmit power issues in a WCDMA cellular system," 3G Mobile Communication Technologies, 2002 Conference Publication No. 489, pp. 244-249, May 2002.

[12] L. Zheng and D. N. C. Tse, "Communication on the Grassmann manifold: a geometric approach to the noncoherent multiple-antenna channel," IEEE Trans. Inf. Theory, vol. 48, no. 2, pp. 359-383, 2002.

[13] T. L. Marzetta and B. M. Hochwald, "Capacity of a mobile multipleantenna communication link in Rayleigh flat fading," IEEE Trans. Inf. Theory, vol. 45, no. 1, pp. 139-157, 1999.

[14] Y.-P. E. Wang and T. Ottosson, "Cell search in W-CDMA," IEEE J. Sel. Areas Commun., vol. 18, no. 8, pp. 1470-1482, 2000. 